Décadrages Décadrages

cinéma, à travers champs Cinéma, à travers champs

$34-36 \mid 2017$

Cinéma de re-montage

\title{
Le « montage des idées", ou comment Joris Ivens et Henri Storck ont appris à parfaire l'art du remontage
}

\section{Bert Hogenkamp}

Traducteur : Sylvain Portmann

\section{OpenEdition}

Journals

Édition électronique

URL : https://journals.openedition.org/decadrages/1029

DOI : 10.4000/decadrages. 1029

ISSN : 2297-5977

\section{Éditeur}

Association Décadrages

\section{Édition imprimée}

Date de publication : 1 mai 2017

Pagination : 26-39

ISBN : 978-2-9700963-3-7

ISSN : 2235-7823

Référence électronique

Bert Hogenkamp, "Le « montage des idées », ou comment Joris Ivens et Henri Storck ont appris à parfaire l'art du remontage », Décadrages [En ligne], 34-36 | 2017, mis en ligne le 19 août 2019, consulté le 04 avril 2022. URL : http://journals.openedition.org/decadrages/1029; DOI : https:// doi.org/10.4000/decadrages.1029 
Bert Hogenkamp

(traduction de l'anglais par Sylvain Portmann)

\section{Le " montage des idées ", ou comment Joris Ivens et Henri Storck ont appris à parfaire l'art du remontage}

1 Le film est également connu sous le

Misère au Borinage (Belgique, I934), film documentaire coréalisé par Joris Ivens et Henri Storck à la suite de la grève des mineurs dans le Borinage, cette région belge du charbon, s'ouvre sur une séquence de montage accusant la «crise dans le monde capitaliste» 1 . Pour cette séquence, les réalisateurs ont utilisé des plans provenant de sources très variées. Certains plans ont été tournés par Ivens et Storck tandis que d'autres sont empruntés à divers films d'actualités. Ces derniers comprennent des et Henri Storck (éd.), «Le Borinage: La grève des mineurs de 1932 et le film de Joris Ivens et Henri Storck», Revue Belge du Cinéma, $\mathrm{n}^{\circ}$ 6-7, hiver i983printemps i984.

Paul Hennebert et Joris Ivens (tenant une caméra Kinamo), photographie du tournage de Misère au Borinage (@ Willy Kessels)

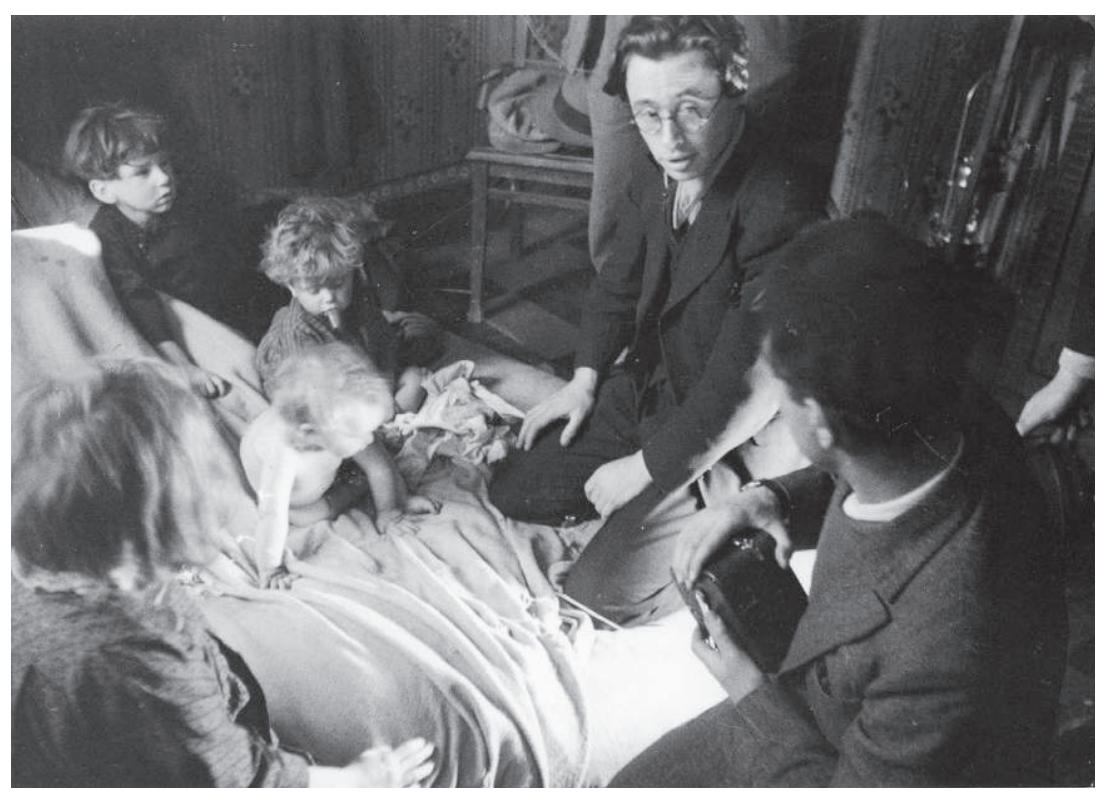


images qui ont déjà été utilisées par Ivens pour son film Nieuwe Gronden (Nouvelle Terre, Pays-Bas, I933). A ceux-là s'ajoute une actualité retirée de la circulation par la compagnie (américaine) qui l'a produite. Un plan utilisé dans la séquence d'ouverture est ainsi doté d'une signification totalement différente, inversant son contexte d'origine. Cette approche recherchée, qu'Ivens appelle le «montage des idées» dans son autobiographie The Camera and $I^{2}$, résulte de plusieurs expériences antérieures de re-montage menées par le Hollandais ainsi que par son collègue belge. Avant d'analyser plus attentivement Misère au Borinage, rappelons la manière dont les deux réalisateurs ont acquis leurs compétences.

Le passé de Joris Ivens (I898-I989) et celui de Henri Storck (I907I999) sont à maints égards comparables. Tous deux ont grandi dans des villes de province (Nijmegen pour Ivens et Ostende pour Storck) et sont fils de commerçants prospères (respectivement patrons de la chaîne de magasins de photographie CAPI et d'un magasin de chaussures). Dès leur jeune âge, ils sont en contact avec les milieux de l'art et des artistes. Ivens par le biais de Jan Toorop et Henry Luyten, tous deux des protégés de son père C. A. P. Ivens, tandis que Storck fréquente les studios de James Ensor, Constant Permeke et Léon Spilliaert qui résident dans des hôtels de la côte à Ostende. Mais c'est le nouvel art du $\mathrm{xx}^{\mathrm{e}}$ siècle, le cinéma, qui captivera Ivens et Storck. Habitant des pays où l'industrie du cinéma n'est pas implantée, ils font leur entrée dans le monde de la cinématographie à travers le phénomène des ciné-clubs. Apparaissant à travers toute l'Europe, les ciné-clubs proposent un ensemble de films qui sont soit ignorés des exploitants ordinaires car jugés pas assez «rentables», soit mis au ban par la censure pour des raisons politiques ou morales, quand ils ne sont pas tout simplement tombés dans l'oubli. Ivens a joué un rôle clé dans la fondation et l'exploitation de la Filmliga à Amsterdam, alors que Storck a fondé un ciné-club dans sa ville natale, Ostende.

Les projections de ces ciné-clubs ont une fonction d'apprentissage pour de jeunes cinéastes en devenir tels qu’Ivens et Storck. La découverte de films soviétiques, allemands, français ou autres (le plus souvent d'avant-garde) leur enseigne les potentialités expressives offertes par l'ombre et la lumière, les angles de caméra insolites et la mise en scène calculée. Mais ce qui les attire le plus, c'est sans aucun doute les possibilités illimitées offertes par le montage, en particulier dans le
2 Joris Ivens, The Camera and I, Berlin, Seven Seas Publisher, 1969, p. 96. 
3 Léon Moussinac est critique de films à L'Humanité; il a notamment publié Le Cinéma soviétique (Paris, Gallimard, I928). Béla Balász fournit régulièrement des articles à Die Weltbühne. Son ouvrage Der Geist des Films (Halle [Saale], Verlag Wilhelm Knapp, I930) constitue la somme de ses réflexions sur le cinéma.

4 J. Ivens, The Camera and I, p. 96 [ma traduction, ainsi que les suivantes]. cinéma soviétique avec des figures emblématiques telles qu’Eisenstein et Poudovkine. La traduction de leurs manifestes et de leurs réflexions théoriques dans des revues de cinéma européennes telles que Filmliga ou Close-Up suscite de nombreux échos. Des critiques bien informés comme Léon Moussinac ou Béla Balász jouent également un rôle important en analysant dans leurs articles et leurs livres les concepts du montage soviétique ${ }^{3}$.

Le premier film d'Ivens, Le Pont (1928), une étude en mouvement sur un pont ferroviaire à Rotterdam, est projeté pour la première fois lors d'une soirée organisée par la section d'Amsterdam de la Filmliga. Son film suivant, Les Brisants (1929; coréalisé avec Mannus Franken), lui permet de se familiariser avec l'art de la narration et celui de la direction d'acteurs non professionnels. La Pluie (ı29; coréalisé avec Mannus Franken) présente une interprétation personnelle et poétique du modèle populaire de la «symphonie urbaine» (le film exemplaire de Walter Ruttmann, Berlin: Symphonie d'une grande ville, a eu un grand impact aux Pays Bas), montrant Amsterdam avant, pendant et après une averse. Ivens a lui-même financé ces films, bénéficiant de sa position de gérant de la filiale d'Amsterdam de la CAPI, qui lui donne un accès facilité aux caméras et au matériel de montage. Son projet de film suivant a été en revanche financé par l'Union générale néerlandaise des travailleurs du bâtiment. En résulte un long documentaire, Nous bâtissons (I930), et une série de courts métrages parmi lesquels Zuiderzee est de loin le plus important.

Tout comme Ivens, Storck est un adepte de la Kinamo, une caméra $35 \mathrm{~mm}$ portative à ressort, qui permet une grande liberté de mouvement, même si la durée des plans tournés est très limitée. Sa ville natale lui offre le sujet d'un de ses premiers films, Images d'Ostende (1929). C'est un nouvel exemple d'une «symphonie urbaine». Storck devient ainsi le «cinégraphiste officiel» de la ville d'Ostende. Comme Ivens, il réalise un essai narratif avec son court métrage de fiction, Idylle à la plage (I93i).

Ivens est le premier à faire l'expérience du montage de bandes d'actualités (que l'on désigne également sous l'appellation de found footage). Plus tard, il se souviendra avoir été «chargé de constituer des programmes de films destinés à des matinées dominicales culturelles et éducatives destinées aux ouvriers ${ }^{4}$. Il s'agit de réunions de la Vereeniging voor VolksCultur (Association pour la Culture Populaire, connue sous l'acro- 
nyme VVVC). C'est une soi-disant «organisation de masse» qui a été fondée en janvier 1928 par le Parti communiste hollandais «pour faciliter l'organisation de séances de films, de spectacles de cabaret et autres attractions, de sorte à limiter au maximum toute interférence avec les autorités qui ne manifestent aucune bienveillance à notre égard ${ }^{5}$. En I929, Ivens réalise un court métrage pour le VVVC, Arme Drenthe (Pauvre Drenthe), qui montre la visite de Louis Visser, membre du Parti communiste, dans la province de Drenthe, région de la tourbe frappée par la misère ${ }^{6}$.

C'est à son retour d'un voyage en Union soviétique (c'est lors de la visite de Poudovkine à Amsterdam en 1929 qu'Ivens est personnellement invité par le réalisateur soviétique à présenter ses films dans la République des paysans et des ouvriers) qu'il s'est mis à collaborer plus étroitement avec le VVVC. Le 28 septembre I930, le VVVC présente sa propre production d'actualités au sein d'un programme qui comporte par ailleurs le film soviétique interdit Prostitution (Oleg Frelich, Prostitutka, 1927) et des chansons militantes chantées par un fameux acteur néerlandais. Selon le journal communiste De Tribune, ces actualités «ne sont finalement rien d'autre que des actualités bourgeoises qui pour l'occasion ont été quelque peu remontées et auxquelles on a ajouté de nouveaux intertitres». Le journal ajoute que «ça a frappé juste. Il est devenu clair pour les spectateurs présents comment de tels «sujets d'actualité à l'écran`, diffusés chaque semaine dans tous les cinémas, doivent être perçus ${ }^{7}$.

Ivens se remémore dans son autobiographie que l'assemblage de ces actualités n'est pas aussi aisé que ne le laisse entendre De Tribune: «Le vendredi soir, nous empruntions quelques actualités commerciales. Le samedi, nous étudions le contenu de ces actualités au regard de la situation internationale et nationale de la semaine; nous les remontions avec d'autres bandes que nous avions à disposition, les dotant ainsi d'une signification politique claire, ajoutant de nouveaux intertitres (les films étaient encore muets) qui explicitaient les relations entre des événements dont les agences d'actualités n'auraient jamais soupçonné l'existence, et qui les auraient très certainement choqués s'ils avaient vu notre utilisation de leur matériel «innocent`». Ivens ajoute: «A la suite de notre séance du samedi matin, nous démontions à nouveau les bandes, pour restituer leur agencement initial avant de les retourner aux agences d'actualités
5 De Tribune, 28 janvier 1928.

6 Le film est également connu sous le titre de De Nood in de Drentsche Venen (La Misère aux tourbières de Drenthe). Le film n'a pas survécu mais un compte rendu de la visite de Louis de Visser, illustré par des photos et des dessins de Henri Pieck, est publié dans l'hebdomadaire illustré Het Leven (2 mars I929). Durant cette visite, le membre du Parti communiste n'est pas seulement accompagné par Pieck, Ivens et par le photographe de Het Leven mais également par Leo van Lakerveld, secrétaire du VVVC et de la section néerlandaise du Secours Rouge.

7 De Tribune, 30 septembre I930. 
8 J. Ivens, The Camera and I, op. cit., p. 97 .

9 Durant le printemps i93I, la VVVC a des sections à Amsterdam, Apeldoorn, Arnhem, Groningen, Haarlem, Leeuwarden et Utrecht.

10 En effet, les deux premiers numéros des actualités du VVVC sont soumis à la commission de censure du film néerlandais avec comme titres Notes filmiques d'Union soviétique (visa de censure $\mathrm{n}^{\circ}{ }_{10784}$ ) et Manifestation de solidarité prolétarienne ( ${ }^{\circ}$ I0995). Le VVVC a manifestement l'intention de les montrer à un public plus large que celui des membres du VVVC, car sinon ils n'auraient pas pris la peine de les soumettre à la commission de censure ni d'en payer les coûts. Voir à ce sujet la Centrale Commissie voor de Filmkeuring aux Archives Nationales à La Haye.

11 De Tribune, i8 novembre i930. Le journal signale que les plans du Sud de l'Union soviétique ont été «tournés par un ami du VVVC en Union soviétique», très probablement Joris Ivens lui-même.

12 De Sovjet-Vriend, $\mathrm{n}^{\circ}$ 2-3, mars I93I, p. 5 .

13 De Tribune, 17 février i93I. qui n'en n'ont jamais rien su» ${ }^{8}$. Tandis qu'Ivens donne l'impression qu'il a agi ainsi à de nombreuses reprises, la presse communiste fournit des informations divergentes. Inspiré par les idées de Willi Münzenberg sur la propagande culturelle, le secrétaire du VVVC Leo van Lakerveld est très favorable à des actualités indépendantes observant l'information du point de vue de la classe ouvrière. Mais il est crucial qu'elles ne soient pas seulement accessibles pour la projection unique de la section d'Amsterdam du VVVC (sans aucun doute la plus importante); il faut encore qu'elles soient mises à disposition des autres sections ${ }^{9}$. Ce qui n'est vraisemblablement pas possible si les bandes doivent être restituées après chaque projection, de la façon décrite par Ivens dans son autobiographie. Ainsi, un certain nombre d'actualités sont remontées de façon permanente, composées de matériel qui ne devait pas être retourné après chaque projection ${ }^{10}$.

Entre novembre I930 et mars I93I, cinq actualités numérotées sont produites par le «collectif [anonyme] du film VVVC», dont Joris Ivens est incontestablement le membre le plus important. Puisqu'aucune de ces actualités n'a survécu, seuls les comptes rendus de la presse peuvent nous renseigner sur leurs contenus. La première actualité du VVVC consiste en un «subtil choix d'images du sud de l'Union soviétique, de Bakou, Kharkov, Kiev, explicitées par d'éloquents intertitres et fragments d'un film plus ancien dans lequel apparaissent Lénine et Staline «en action〉 et plus tard une magnifique suite d'images montrant comment les camarades russes célèbrent leur Octobre " ${ }^{11}$. Le numéro suivant «est dévolu [...] au travail du Secours Rouge. Au centre, se trouvent des plans tournés par le collectif du film VVVC lors d'un tour de prospection et de récolte de fonds mené à Jordaan, dans le district d'Amsterdam, et montés avec d'anciennes images». Le contenu du troisième numéro est résumé ainsi: «La terreur en Europe et en Amérique; Vie, travail et mort de Lénine; Construction en Union soviétique» ${ }^{12}$. Le quatrième, La Face de deux mondes, «force les spectateurs à comparer les résultats de la révolution allemande, menée par les sociaux-patriotes et les sociaux-fascistes, avec la Révolution russe menée par les bolchéviques » ${ }^{13}$. Le cinquième et dernier numéro, d'une durée de deux bobines, est de loin le plus ambitieux. Intitulé L'Intervention est proche, on y montre «la guerre mondiale, la trahison des sociaux-démocrates et la résistance des masses», suivi par «la construction au sein de l'Union soviétique et la préparation des impéria- 
listes à l'intervention». Figurent également des plans tournés par le collectif VVVC de «la manifestation du Secours Rouge du dimanche 8 mars [à Amsterdam, note de l'auteur] et des attaques perpétrées par la police contre les manifestants» ${ }^{14}$.

La série d'actualités du VVVC s'interrompt au cinquième numéro. C'est la conséquence de la refonte au printemps i93 du VVVC en une Association des Amis de l'Union soviétique (Vereeniging van Vrienden der Sovjet-Unie, VVSU) qui se concentre spécifiquement sur la présentation de produits culturels (comprenant les actualités) provenant d'Union soviétique. De plus, la présence stimulante d'Ivens est vivement regrettée, celui-ci ayant été invité à réaliser un film en Union soviétique sur la Construction socialiste à Magnitogorsk (Le Chant des Héros, 1933).

Les comptes rendus dans la presse montrent clairement que les cinq actualités du VVVC reposent essentiellement sur des «parallèles implacables » [deadly parallels ${ }^{15}$, par exemple sur l'opposition entre le «bon» (Révolution russe, construction en Union soviétique) et le «mauvais» (Révolution allemande, terreur en Europe et en Amérique); entre «nous» (manifestants du Secours Rouge) et «eux» (la police); entre «nos» films (tournés par «un ami du VVVC» ou par le collectif du VVVC) et les «leurs» (provenant d'autres sources). Les actualités comprennent des plans qui sont parfois explicitement identifiés comme «anciens», que nous désignerions aujourd'hui comme des «films d'archives».

Les intertitres «éloquents» conçus pour les actualités du VVVC jouent un rôle important, consistant à mettre en avant l'interprétation communiste des événements montrés. Puisque les bandes sont muettes, il faut concevoir un accompagnement musical. A Amsterdam, l'association a à disposition son propre «orchestre collectif du VVVC», dirigé par le compositeur professionnel Johan Voehrs; ailleurs, ils doivent trouver d'autres solutions ${ }^{16}$. Voehrs s'est pourtant rendu à l'occasion dans les villes d'autres sections du VVVC. Au Cinéma Palace d'Utrecht, il doit par exemple s'accommoder d'un piano complètement désaccordé ${ }^{17}$. A d'autres occasions, des disques de gramophone offrent d'« excellentes » alternatives ${ }^{18}$.

Les premières expériences de re-montage de Henri Storck sont amorcées suite à une remarque de son collègue belge Charles Dekeukeleire: «Storck ne sait pas monter. Il fait des films intéressants, mais ce n'est pas un maître du montage ${ }^{19}$. En 1932, alors qu'il vit grâce à l'exploitation
14 De Tribune, 24 mars I93I.

15 Au sujet de la notion de "parallèle implacable» [deadly parallel], voir Bert Hogenkamp, Deadly Parallels. Film and the Left in Britain, 1929-39, Londres, Lawrence \& Wishart, I986, pp. I6-I7.

16 Voir Edith van der Heijde, Dissonante geluiden en sprekende filmvertoningen. Een vergelijkend cultuurhistorisch onderzoek naar de houding van de Nederlandsche Filmliga, de Vereniging voor Onderwijs-en Ontwikkelingsfilms en de Vereniging voor Volkscultuur ten opzichte van filmmuziek en de introductie van de geluidsfilm in Nederland, Mémoire de Maîtrise, Université d’Utrecht, 20II, pp. 29-35 (http://dspace.library.uu.nl/ handle/I874/2I7I8I).

17 De Tribune, 23 décembre 1930.

18 De Tribune, 24 décembre 1930.

19 Henri Storck, entretien avec l'auteur, Bruxelles, 7 février I977; l'entretien a été publié dans Skrien, $\mathrm{n}^{\circ} 66$, juilletaoût I977, pp. 25-27. 
20 Henri Storck, entretien avec l'auteur, Bruxelles, 7 février 1977 .

21 Les scénarios de films non réalisés et recueillis dans La Courte échelle et autres scénarios (La Louvière, Le Daily-Bul, I995) illustrent bien jusqu'à quel point le surréalisme l'a influencé au début des années I930.

22 Ce ne fut que durant les années i96o que des versions sonores de ces films furent produites à partir des disques de gramophone que Storck avait choisis en I932 pour les versions muettes. d'un laboratoire artisanal de films à domicile, Storck est amené à examiner l'intégralité des actualités Eclair de 1928, 52 bobines au total, et d'en isoler les éléments en lien avec l'athlétisme pour le compte de la Fédération Belge d'Athlétisme. Il exige en retour le droit d'utiliser à sa guise le reste du matériel. Storck a l'intention de monter un ou deux courts métrages à partir de ces bandes et d'ainsi prouver que Dekeukeleire avait tort. A la différence d'Ivens et des actualités du VVVC, Storck n'a pas l'intention d'énoncer un message politique clair. Mais ce qui le frappe, ce sont - dix ans après la fin de la Première Guerre mondiale - les signes évidents de réarmement au sein d'un grand nombre d'actualités, et ceci malgré le fait que le Pacte Briand-Kellogg est signé en I928, décrétant la guerre hors-la-loi. Storck décide ainsi de prendre pour thème d'un de ses films «le soldat inconnu, la victime de toujours, l'homme de la rue qui souffre éternellement» ${ }^{20}$. Dans L'Histoire du soldat inconnu, des parades militaires et des processions religieuses ainsi que l'abdication du tsar russe en I9I7 (qu’Eclair a ressorti de ses archives et inséré dans une actualité de 1928 à l'occasion de la mort du neveu du tsar à Nice) contrastent avec les images des cheminées d'usine se dissipant au ralenti et de l'exhumation du corps d'un «soldat inconnu».

Le second film, Sur les bords de la caméra, manifeste encore plus clairement l'empreinte du surréalisme sur Storck, mouvement qui exerce par ailleurs une grande influence sur l'art belge de l'époque ${ }^{21}$. Storck intercale par exemple un plan de femmes gymnastes jouant en rythme à la balle au sein d'une série de plans d'incendies spectaculaires. Ajoutons que Storck a également inclus des séquences qui n’ont pas été filmées par Eclair, mais qui sont tirées du court métrage Blutmai 1929 (Phil Jutzi), montrant la confrontation sanglante entre la police et les ouvriers (majoritairement communistes) qui manifestent pour le droit de célébrer la Fête du Travail. Ce film est par ailleurs distribué en Hollande par le VVVC.

Ces deux courts métrages montés, se pose alors la question pour Storck de savoir où les projeter. Bien qu'il ait composé des partitions de musique à partir de disques de gramophone, il lui est difficile de trouver un exploitant désireux de diffuser ces films essentiellement muets - et plutôt inhabituels de par leur montage et par leur sujet, et de plus dépourvus du moindre intertitre qui aurait permis d'expliquer les événements présentés à l'écran ${ }^{22}$. Storck tente sa chance en France et soumet L'Histoire du soldat inconnu à la censure française. Pourtant, le véné- 
rable M. Edmond Sée refuse de lui accorder un visa car le film «tourne en dérision l'Armée française ${ }^{23}$. Jean Lods, ami et collègue de Storck, lui suggère d'essayer de le diffuser via la coopérative communiste La Bellevilloise dans le $20^{\mathrm{e}}$ arrondissement de Paris. Le réalisateur assiste à la projection test durant laquelle le film est évalué - et rejeté - par les dirigeants communistes Marcel Cachin et Paul Vaillant-Couturier. Par la suite, Lods dévoilera à Storck leur identité, alors que le réalisateur belge est terrorisé par le fait qu'ils saluent tous les policiers de la ville lors du trajet de retour en voiture vers la Concorde ${ }^{24}$.

Après avoir terminé son film en Union soviétique, Ivens retourne aux Pays Bas au printemps i933. Il commence à travailler sur une nouvelle version, sonore cette fois, de Zuiderzee. Durant son absence, les membres de son équipe (également connue sous le nom de Studio Joris Ivens), qui comprend Willem Bon, Mark Kolthoff, Joop Huisken, John Fernhout et Helen van Dongen, ont continué de filmer la fermeture finale du barrage, le drainage et la réclamation des nouvelles terres. Il demande au compositeur Hanns Eisler d'écrire une partition pour le nouveau film, intitulé Nouvelle Terre. Ivens le conclut avec une séquence de montage qui condamne le système capitaliste. Il soutient que les céréales moissonnées sur la terre nouvellement obtenue (arrachée à la mer) ne sont pas destinées à l'Homme mais à la spéculation. Il illustre cette thèse avec des bandes d'actualités qui montrent notamment des hommes à bord d'un bateau vidant des sacs de céréales dans la mer et d'autres les jetant au feu. Dans The Camera and I, Ivens se souvient non sans fierté de l'argument avancé par le censeur français pour interdire Nouvelle Terre: «c'est trop de réalité» ${ }^{25}$.

Mais revenons à la scène d'ouverture de Borinage. Elle comprend I9 plans. Quatre d'entre eux peuvent être identifiés sans aucun doute comme ayant été tournés par Ivens et Storck. Le plus évident est celui de la famille Mouffe dévorant les pommes de terre apportées par l'équipe du film. Le même plan est répété par la suite ${ }^{26}$. Deux plans documentant la Marche de la Faim, de Lille à Paris, sont fournis par Jean Lods. Le reste provient de bandes d'actualités. A deux reprises, il est possible d'identifier la source: la Ligue new-yorkaise du Film et de la Photo (New York Film and Photo League). Il s'agit de la section locale d'une organisation nationale de gauche qui s'est fait un nom en filmant la détresse des chômeurs aux Etats-Unis et leur combat pour de meilleures conditions
23 Henri Storck, entretien avec l'auteur, Bruxelles, 7 février 1977.

24 Id.

25 J. Ivens, The Camera and I, op. cit., p. 99 .

26 Ivens emploie le même plan deux décennies plus tard dans la production de la DEFA Le Chant des fleuves (I954) car il symbolise parfaitement pour lui l'idée des «travailleurs qui ont faim». 
de vie. L'origine du plan montrant du lait jeté d'un camion provient d'une actualité commerciale. Il a été acquis par la Ligue new-yorkaise du Film et de la Photo, qui l'a inclus dans son film d'actualités America Today No 2. Contrairement à sa signification dans Borinage (et auparavant dans Nouvelle Terre), il montre en réalité des paysans du lait en grève dans le Wisconsin qui ont intercepté un chargement visant à briser la grève et qui s'assurent en le déversant qu'elle soit maintenue.

Misère au Borinage (Joris Ivens et Henri Storck, 1934)
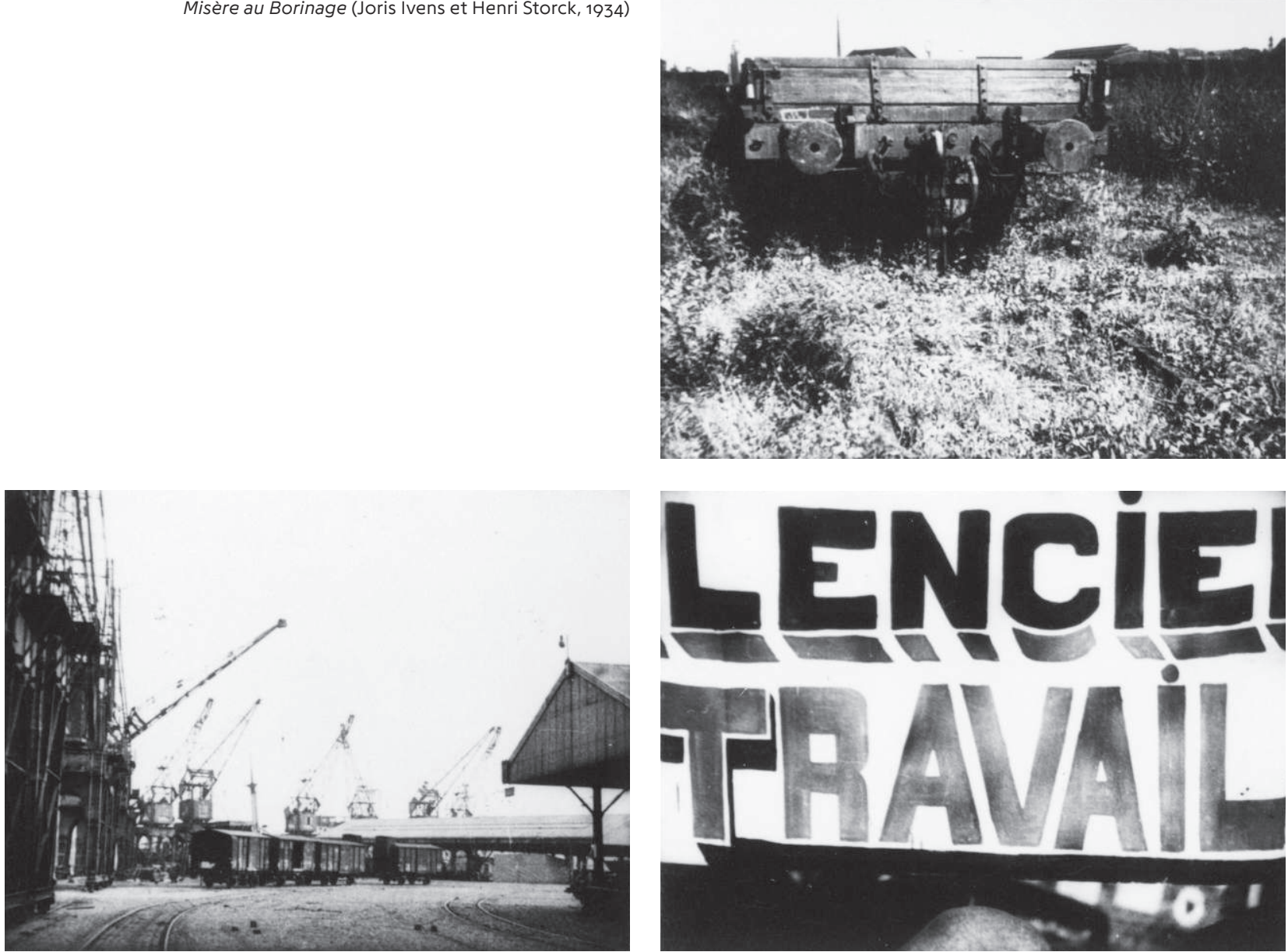
Le massacre d'ouvriers métallurgistes en grève et non armés perpétré par des miliciens à Ambridge, en Pennsylvanie, le 4 octobre i933, constitue un autre élément issu de America Today No 2. La confrontation a été filmée par un opérateur de RKO-Pathé et a été exploitée commercialement. Elle a généré un phénomène médiatique au détriment de RKOPathé qui l'a donc rapidement retirée de la circulation ${ }^{27}$. Cet élément est décrit par Samuel Brody, critique de cinéma pour le Daily Worker, comme
27 Russell Drummond Campbell, $R a$ dical Cinema in the United States, I9301942: the Work of the Film and Photo League, Nykino, and Frontier Films, Thèse de doctorat, Evanston, Northwestern University, 1978 , p. I22.
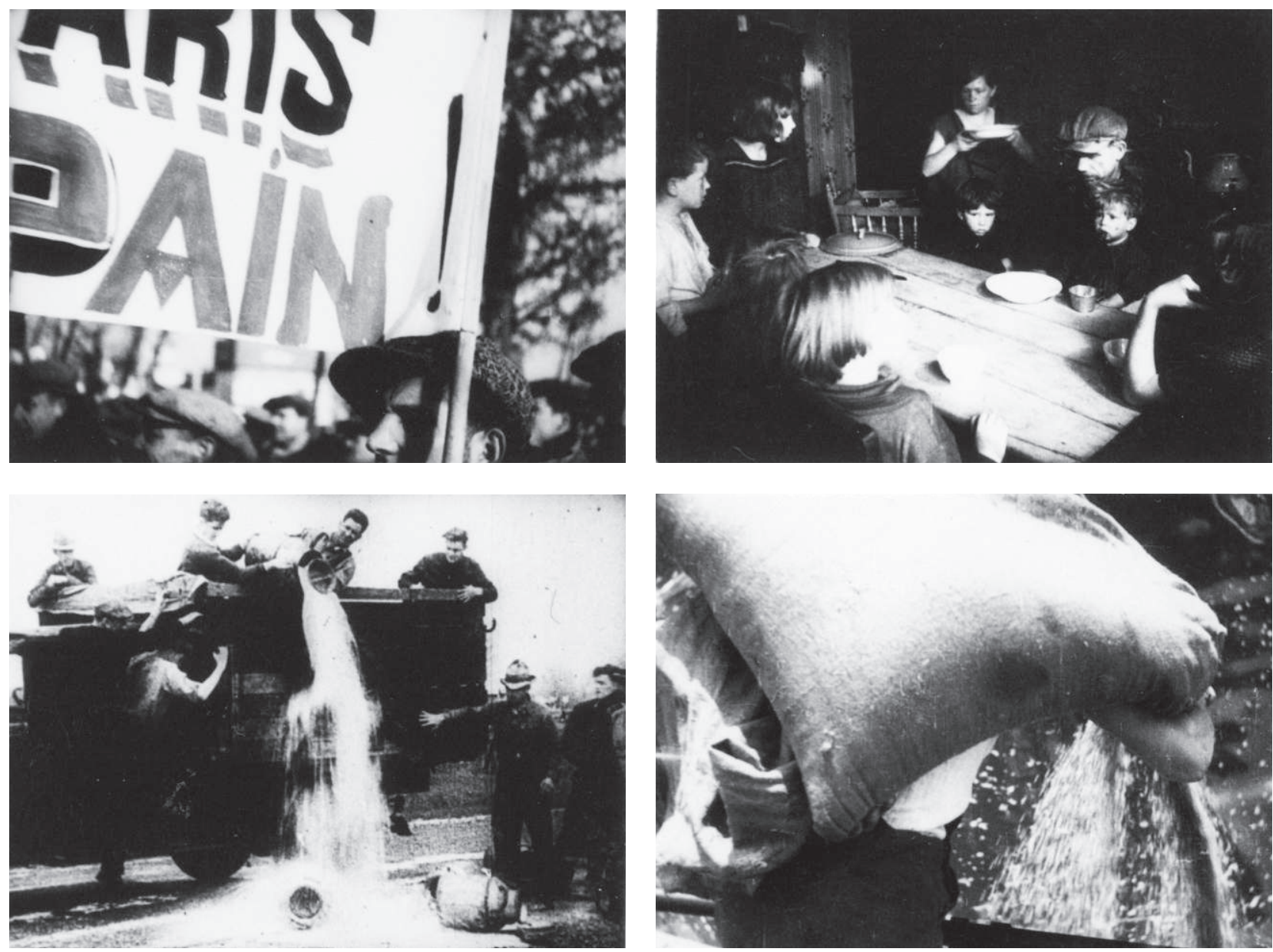
28 «Lens», «Flashes and Close-Ups», dans Daily Worker, 20 octobre I933, cité par Russell Drummond Campbell, op. cit. la preuve ultime de l'adage du romancier français Henri Barbusse: «de nos jours, la vérité est révolutionnaire». La Ligue new-yorkaise du Film et de la Photo a donc voulu en obtenir une copie ${ }^{28}$. Comment ce plan trouva son chemin jusqu'en Europe de l'Ouest demeure inconnu, mais il a dû y avoir des échanges réguliers, puisque America Today No 2 comprend également un plan sur la manifestation du Front Uni à Paris le ı2 février I934, que la Ligue new-yorkaise du Film et de la Photo n’a
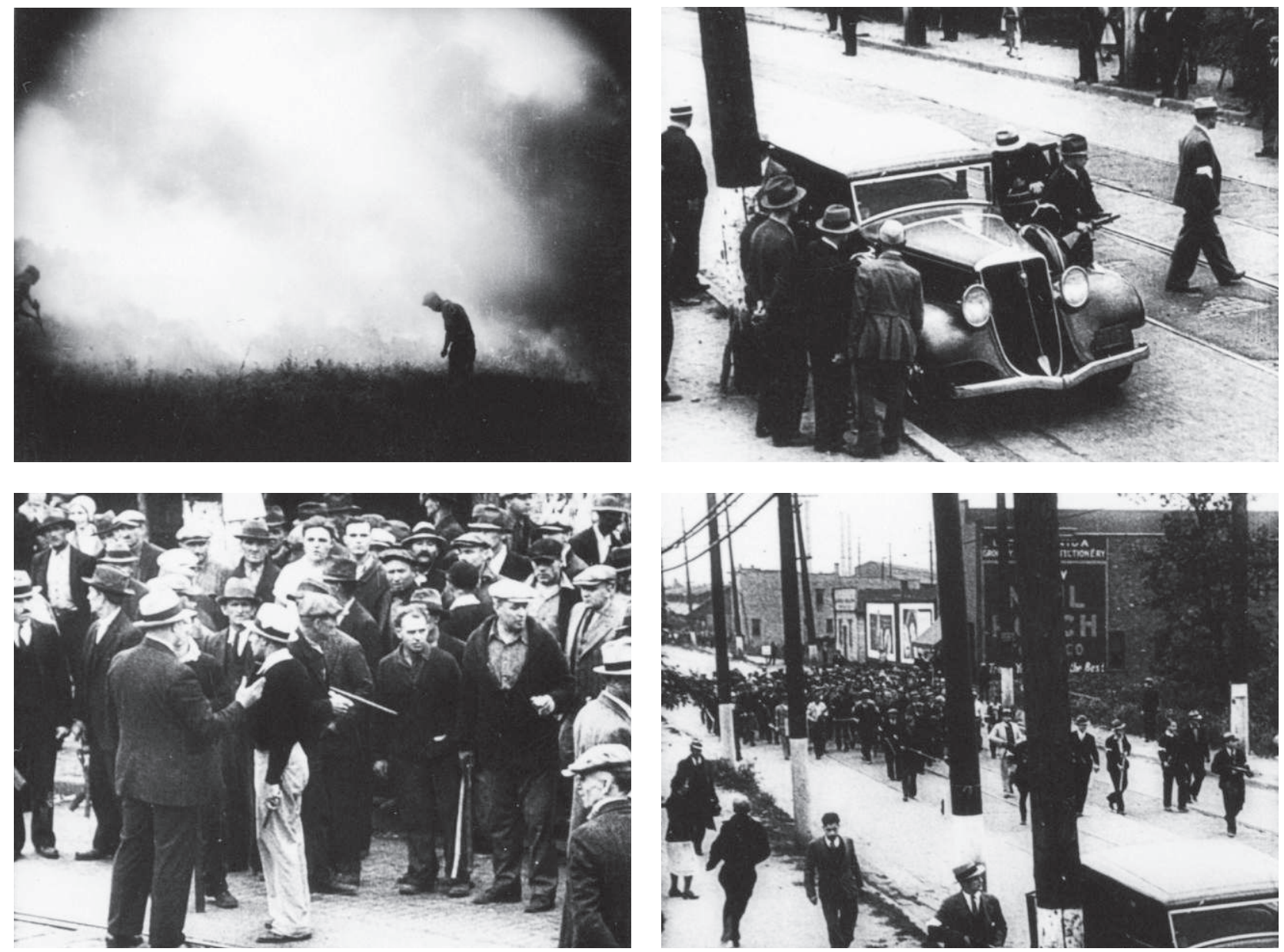
vraisemblablement pas filmé elle-même mais qu'elle a dû obtenir par le biais d'une source française. Dans Borinage, Ivens et Storck utilisent la plupart des actualités d'Ambridge (dix plans différents), car il leur importe d'ancrer leur thèse dès les neuf premiers plans du film: notamment que le capitalisme est en crise, les images parlant d'elles-mêmes. C'est pour cette raison qu'ils n'utilisent que des intertitres descriptifs, «neutres».
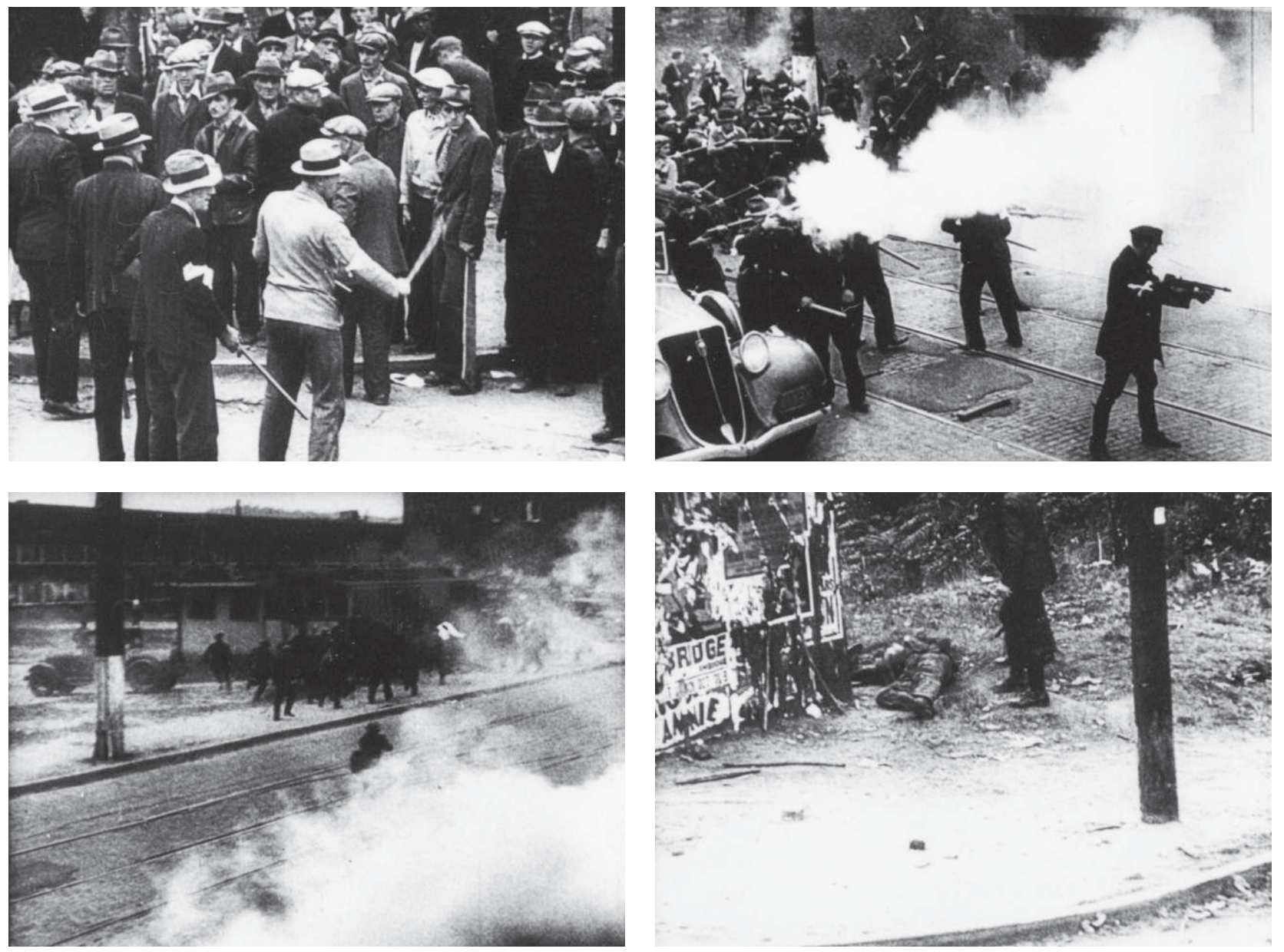
Interrompues par une séquence mettant en scène un jeune mineur subvenant aux besoins de sa mère, veuve, grâce à son maigre salaire de la mine, deux autres séquences de compilation suivent dans lesquelles des bandes d'archive [stock footage] ainsi que du matériel tourné par les réalisateurs sont savamment mélangés. La première séquence analyse la situation économique et sociale du Borinage, tandis que la seconde explique ce qui s'est passé durant la grève des mineurs de i932. Dans
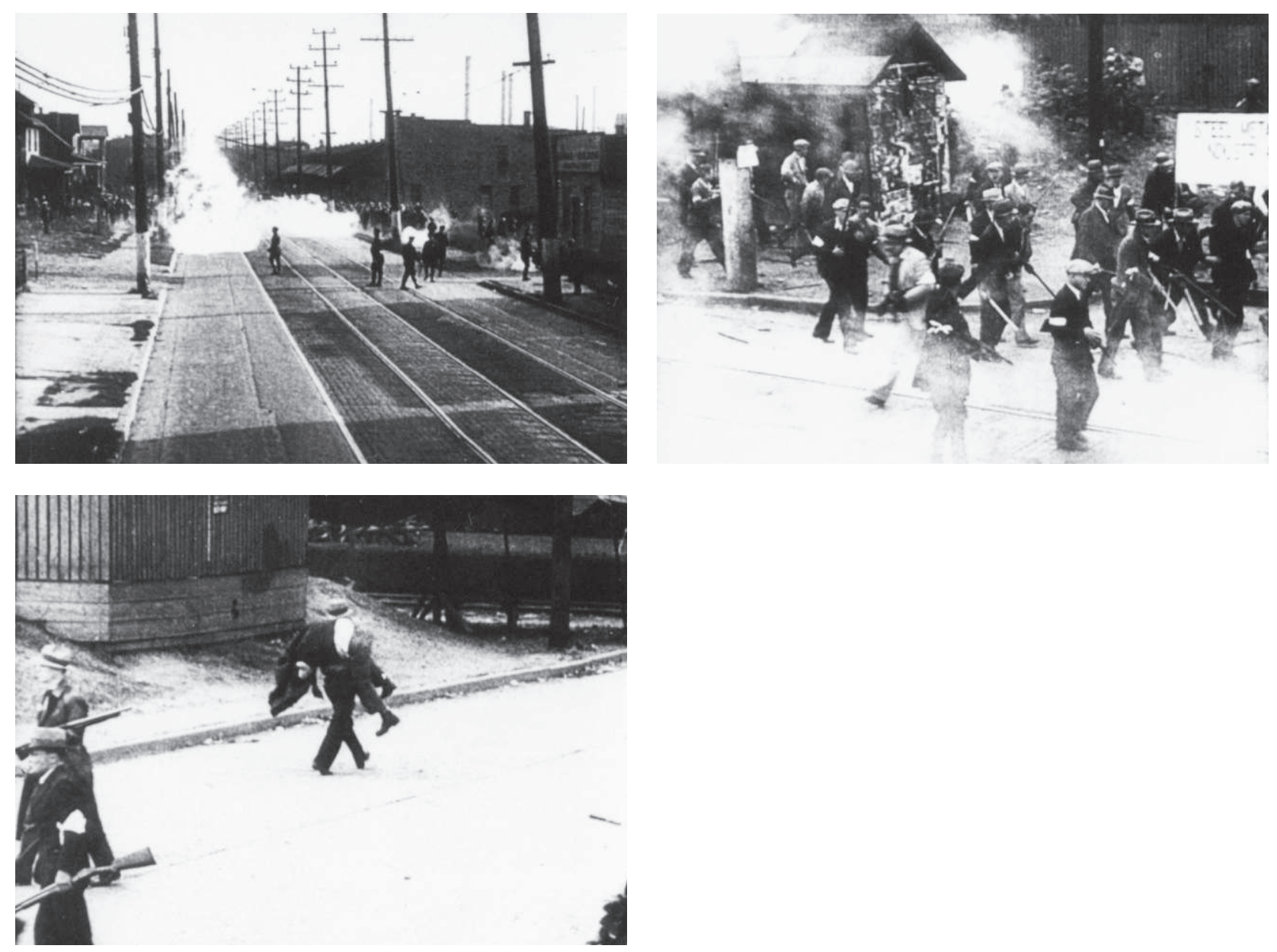
les deux cas, Ivens et Storck ont recours à des intertitres critiques, «subjectifs».

Comment pouvons-nous juger de l'art du «montage des idées» dans Misère au Borinage? Il est évident que la méthode mise en œuvre pour ce film est plus proche de la démarche adoptée par Ivens pour les actualités du VVVC que de celle de Storck pour L'Histoire du soldat inconnu ou Sur les bords de la caméra. Néanmoins, les séquences de montage du film ne se réduisent pas à une simple compilation, telle que définie par William C. Wees dans son ouvrage Recycled Images. The Art and Politics of Found Footage Films ${ }^{29}$. Selon Wees, les principes caractéristiques de la compilation proviennent de «plans tirés de films qui n’ont pas nécessairement de liens entre eux; un concept (thème, argument, histoire) qui motive le choix des plans et l'ordre dans lequel ils apparaissent; et un accompagnement verbal (voix over ou texte sur l'écran ou les deux) qui relie les plans aux concepts ${ }^{30}$. Dans Misère au Borinage, les bandes d'archive (Wees utilise le terme de "found footage») sont habilement mélangées à des plans tournés par les réalisateurs eux-mêmes. C'est une méthode que Wees n'a tout simplement pas pris en considération. Il n'envisage pas non plus l'éventualité qu'un réalisateur puisse inclure des séquences (d'actualités) complètes au sein de son «film de compilation» - aux côtés de plans uniques «qui n’ont pas nécessairement de relation entre eux». Dans Misère au Borinage, c'est le cas avec la séquence Ambridge, que Wees aurait certainement catégorisée comme du collage, sa catégorie favorite, car elle «critique, met au défi et subvertit potentiellement le pouvoir des images, produites et diffusées par les médias dominants » ${ }^{31}$.
29 William C. Wees, The Art and Politics of Found Footage Films, New York City, Anthology Film Archive, 1993 (https:// monoskop.org/images/3/34/Wees_ William_C_Recycled_Images_The_ Art_and_Politics_of_Found_Footage _Films.pdf). 30 Id., p. 35 .

31 Id., p. 33. 